\title{
The Trans-Iron Galactic Element Recorder for the International Space Station (TIGERISS)
}

\section{Brian F. Rauch, ${ }^{\dagger, a, *}$ Nathan E. Walsh ${ }^{a}$ and Wolfgang V. Zober ${ }^{a}$ for the TIGERISS Collaboration}

(a complete list of authors can be found at the end of the proceedings)

${ }^{a}$ Department of Physics and McDonnell Center for the Space Sciences, Washington University, St. Louis, MO 63130 USA

E-mail: brauch@physics.wustl.edu

TIGERISS is an Ultra-Heavy Galactic Cosmic Ray (UHGCR) detector to be proposed to the NASA Astrophysics Pioneers Program capable of measuring the abundance relative to ${ }_{26} \mathrm{Fe}$ of every element from ${ }_{5} \mathrm{~B}$ to ${ }_{82} \mathrm{~Pb}$. It is evolved from the LDB TIGER and SuperTIGER balloon instruments and the Heavy-Nuclei Explorer SMEX, and compared to its predecessors, TIGERISS will have a greatly improved capability to definitively identify UHGCR nuclei. This has been demonstrated in component accelerator tests at CERN, including silicon strip detectors in place of scintillators. The geometry factor for TIGERISS is estimated to be from 1.1 to $1.7 \mathrm{~m}^{2} \mathrm{sr}$ depending on the ISS attachment point, compared to $0.6 \mathrm{~m}^{2} \mathrm{sr}$ for TIGER. Within one-year TIGERISS would observe $\sim 27{ }_{56} \mathrm{Ba}$ nuclei, a $20 \%$ statistically significant result comparable to the current SuperTIGER data set. Not requiring corrections for atmospheric interactions and scintillator saturation effects the TIGERISS results would be cleaner, and they would also make preliminary measurements to higher charges that will test models for cosmic-ray origins and acceleration. TIGERISS will measure UHGCR nuclei resulting from neutron-capture nucleosynthesis in heavy stars, supernovae, and binary neutron-star mergers and will probe the relative contribution of r-process elements to the cosmic rays.

$37^{\text {th }}$ International Cosmic Ray Conference (ICRC 2021)

July 12 th - 23rd, 2021

Online - Berlin, Germany

\footnotetext{
*Presenter

${ }^{\dagger}$ Research supported by the McDonnell Center for the Space Sciences and the Peggy and Steve Fossett Foundation
} 


\section{Introduction}

The Trans-Iron Galactic Element Recorder for the International Space Station (TIGERISS) will investigate two critically important aspects of the grand cycle of matter in the galaxy: the nature of the astrophysical reservoirs of nuclei at the Galactic cosmic-ray (GCR) sources and the mechanisms by which nuclei are removed from the reservoirs and injected into the cosmic-ray accelerators. Evidence from the GCR indicates their source is drawn primarily from older interstellar media (ISM) with admixture of fresh nucleosynthetic products of younger stars, they are accelerated in shock waves from stellar deaths, they help energize Galactic magnetic fields and they feed back into the process of new star formation, as depicted in Fig. 1. This picture has been pieced together over many years from evidence gleaned from measurements of elemental and isotopic composition, energy spectra, and gamma rays emitted in cosmic-ray interactions with the ISM. For many years cosmic-ray acceleration and the r-process neutron capture synthesis of the heavier elements in the cycle shown in Fig. 1 was thought to occur predominantly if not exclusively in supernovae. More recent multi-messenger observations of kilonovae involving gravitational wave [1] and broader electromagnetic spectral observations [2] have provided strong evidence supporting the model of binary neutron star merger synthesis of the heaviest r-process elements. The first single-element resolution ultra-heavy GCR (UHGCR) measurements above ${ }_{40} \mathrm{Zr}$ by SuperTIGER indicate that something is missing from the GCR source (GCRS) model with a significant contribution from OB associations and preferential acceleration of elements condensed on dust grains [3, 4]. TIGERISS will investigate the cosmic-ray elemental composition of the heavier elements with unprecedented resolution to address these scientific questions.

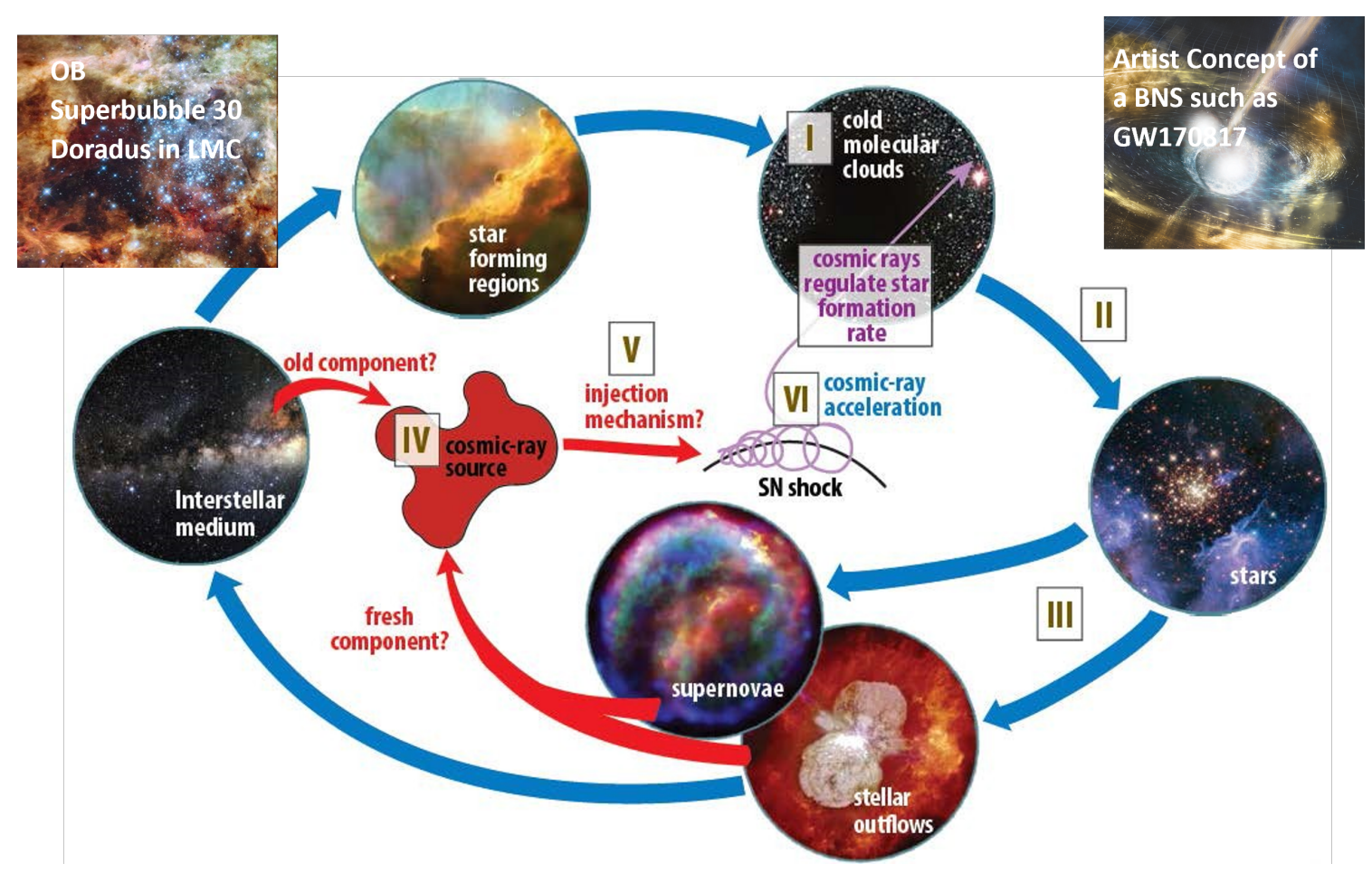

Figure 1: Cosmic cycle of synthesis and distribution of Galactic matter showing cosmic rays as both a messenger of fresh nucleosynthetic material and a driver of the cycle. 


\section{TIGERISS Instrument}

TIGERISS is an evolutionary development of the TIGER family of instruments, and improves on the exceptionally successful SuperTIGER with better charge resolution and dynamic range. TIGERISS utilizes three basic detector subsystems to unambiguously measure the charge of all GCR from ${ }_{5} \mathrm{~B}$ to ${ }_{22} \mathrm{~Pb}$ with energies greater than $\sim 0.2 \mathrm{GeV} /$ nucleon. Two crossed layers of silicon strip detectors (SSD) at the top and bottom of the instrument measure particle trajectories and ionization energy deposits $(d E / d x)$. Two Cherenkov detectors measure nuclear charge $(Z)$ and velocity $(\beta)$ : C1 with an acrylic radiator (optical index of refraction $n=1.49$ ) and C0 with a silica aerogel radiator $(n=1.04)$. Figure 2a below shows the TIGERISS instrument model for the Japanese Experiment Module "Kibo" Exposed Facility (JEM-EF) configuration, and Fig. 2b with it mounted in a JEM-EF pallet.
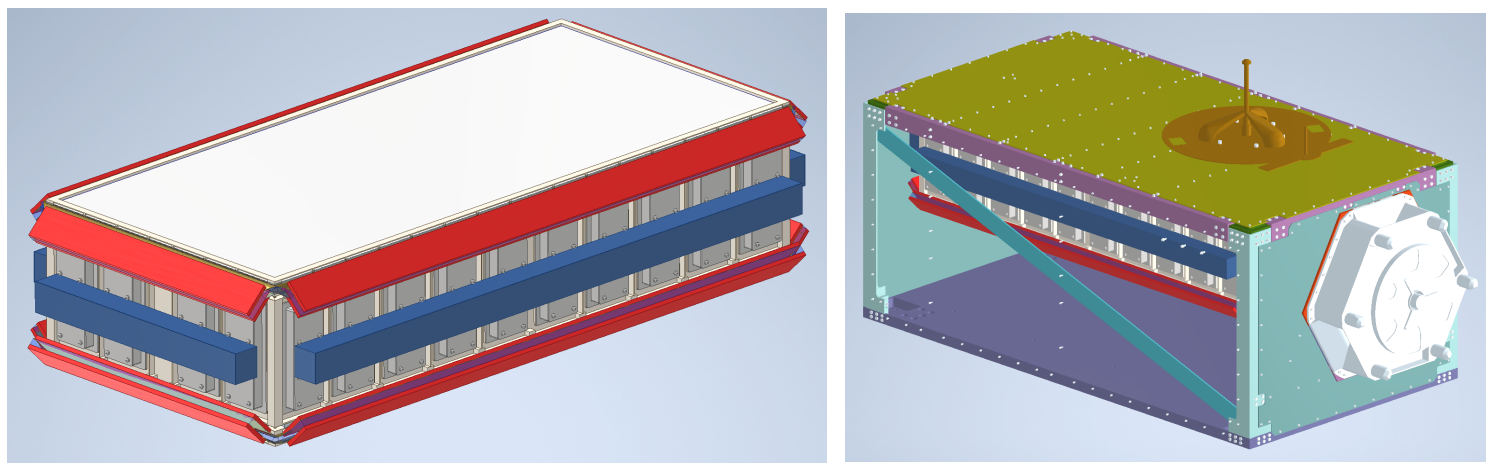

Figure 2(a): Technical model of TIGERISS detector stack with Figure 2(b): TIGERISS instrument model shown top and bottom crossed SSD layers between aerogel and acrylic mounted in the JEM-EF pallet. Cherenkov detectors.

The TIGERISS design draws on the heritage of TIGER/SuperTIGER, HEAO-3, Parker Solar Probe, and STEREO. The SSD detectors flown on these last two instruments provide the ability to measure nuclei $Z \geq 5$ with single element resolution down to $\sim 200 \mathrm{MeV} /$ nucleon, which has been proven in accelerator tests [5] with excellent charge resolution $\left(\sigma_{Q} \leq 0.24\right)$. These detectors are a significant improvement on the scintillators used in TIGER/SuperTIGER [6, 7] in both resolution and signal response with $Z$ since they do not suffer from saturation effects. The charge assignment method using the multiple SSD $d E / d x$ measurements independently and with Cherenkov signals above their thresholds complements the acrylic Cherenkov ( $\mathrm{n}=1.49, \beta>0.67, \mathrm{KE} \geq 325$ $\mathrm{MeV} /$ nucleon) versus aerogel Cherenkov ( $\mathrm{n}=1.04, \beta>0.96, \mathrm{KE} \geq 2.25 \mathrm{GeV} /$ nucleon) technique successfully demonstrated on SuperTIGER/TIGER for energies above the aerogel threshold [6, 7]. The technical measurement range extends to the end of the periodic table, but statistics limit it to $\mathrm{Z} \sim 82$ for the expected possible exposure duration. Readout of both SSD Ohmic- and strip-side charges allows for optimization for different charge ranges for these sides.

The base line TIGERISS instrument is for the JEM-EF, but we can use other ISS accommodation locations as well. Instruments for the European Space Agency Columbus Laboratory external payload $\left(\sim 0.73 \mathrm{~m}^{2}, \sim 1.16 \mathrm{~m}^{2} \mathrm{sr}\right)$ and ExPRESS Logistics Carrier (ELC) $\left(\sim 0.79 \mathrm{~m}^{2}, \sim 1.10\right.$ $\left.\mathrm{m}^{2} \mathrm{sr}\right)$ would be smaller than the JEM-EF configuration $\left(\sim 1.1 \mathrm{~m}^{2}, \sim 1.66 \mathrm{~m}^{2} \mathrm{sr}\right)$ and would have proportionally less exposure. More detail on different TIGERISS geometries and associated geom- 
etry factors is in a paper in this conference documenting the method used to estimate TIGERISS measurements [8]. CALET, on the ISS as a JEM-EF experiment since 2015, has preliminary UHGCR measurements through ${ }_{40} \mathrm{Zr}$ that are consistent with SuperTIGER and ACE-CRIS [9] and demonstrates that the ISS is a good platform for measuring the UHGCR.

\section{Science Objectives}

\subsection{Ultra-Heavy Galactic Cosmic Rays}

The Galactic cosmic rays (GCR) and Solar System (SS) represent two directly measurable samples of Galactic matter, with the GCR being far younger, on the order of millions of years old, and the SS being a $\sim 4.6$ billion year old sample of the Galactic ISM. A comparison of the relative abundances of GCR with energies of $2 \mathrm{GeV} /$ nucleon to those of SS from ${ }_{1} \mathrm{H}$ to ${ }_{40} \mathrm{Zr}$ normalized to ${ }_{14} \mathrm{Si}=1$ is shown in Fig. 3. The major differences between the GCR and SS abundances seen below ${ }_{26} \mathrm{Fe}$ are generally understood to arise from elemental acceleration efficiencies of the GCR and nuclear spallation between the GCR source (GCRS) and detection.

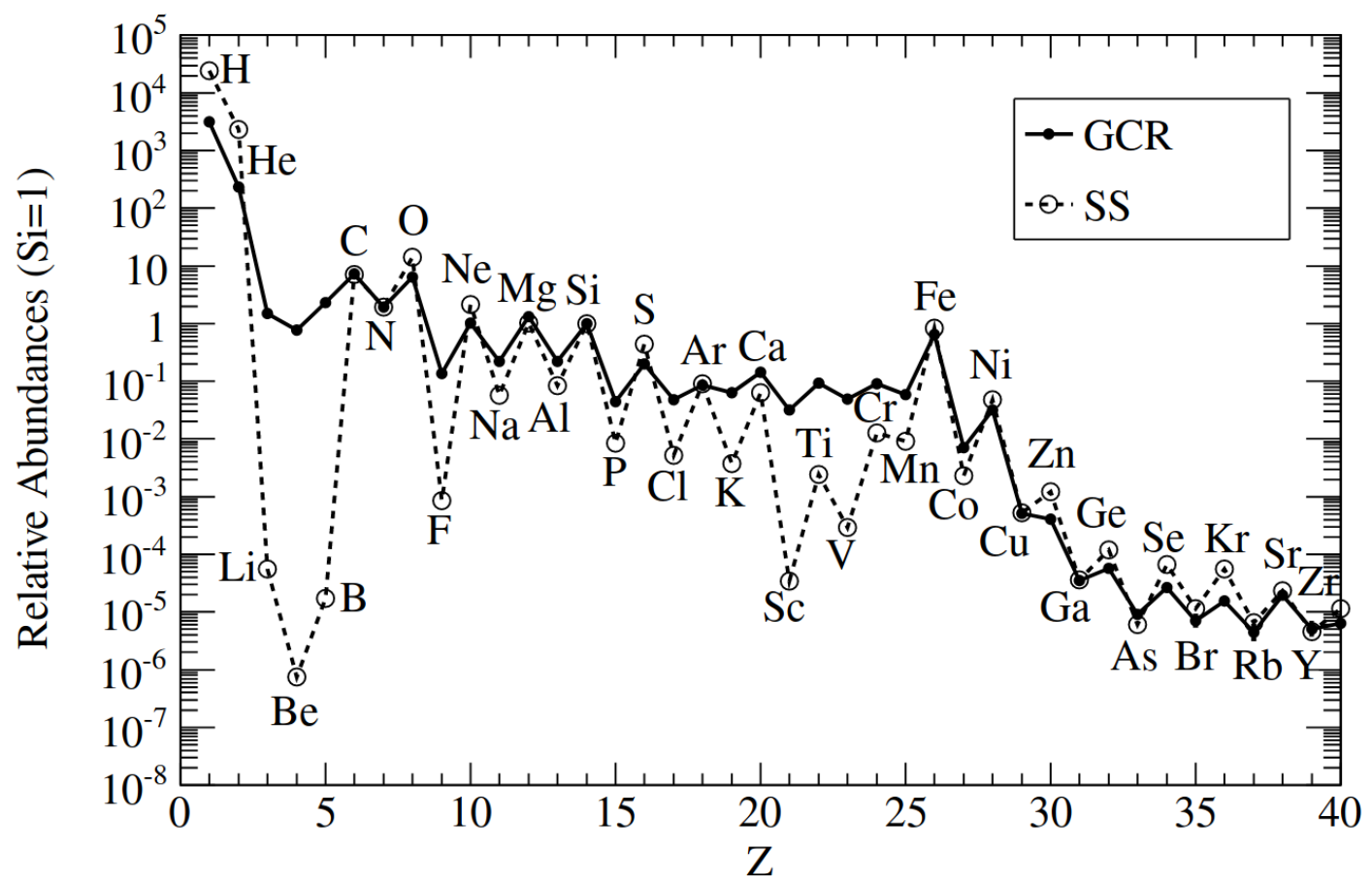

Figure 3: Solar System (SS) [10] and Galactic cosmic-ray (GCR) relative abundances at $2 \mathrm{GeV} /$ nuc. GCR data is sourced for $1 \leq Z \leq 2$ from [11], $Z=3$ from [12], $4 \leq Z \leq 28$ from [13], $Z=29$ from [6], and $28 \leq Z \leq 40$ from [7] and normalized to ${ }_{14} \mathrm{Si}$.

The much rarer ultra-heavy Galactic cosmic rays (UHGCR), ${ }_{30} \mathrm{Zn}$ and higher charge elements, similarly provide clues into the origins of the GCR when compared to SS. Figure 3 shows that in the $\mathrm{GCR}{ }_{26} \mathrm{Fe}$ is $\sim 5 \times 10^{3}$ times less abundant than ${ }_{1} \mathrm{H}$, and that the UHGCR are in turn $\sim 10^{5}$ times less abundant than ${ }_{26} \mathrm{Fe}$. UHGCR measurements with single-element resolution made up to ${ }_{40} \mathrm{Zr}$ by TIGER [6], SuperTIGER [7] and ACE-CRIS [14], which agree within uncertainties, together with 
ACE-CRIS isotopic measurements $\left({ }_{10}^{22} \mathrm{Ne}\right.$ [15], ${ }_{26}^{56} \mathrm{Fe}$ [16]), suggest the GCRS is enhanced in material produced in massive stars ejected by solar winds and in supernovae. The GCR observations up to $40 \mathrm{Zr}$ in Fig. 5 show that refractory elements that are more readily embedded in interstellar dust grains preferentially accelerated over volatile elements, and that injection into the GCR for both refractory and volatile elements appears to follow a charge dependence consistent with their grain sputtering cross sections $\left(\propto Z^{2 / 3}\right)$ [17]. This has been taken as evidence that a significant fraction of the cosmic rays originate in $\mathrm{OB}$ associations where material from earlier generations of massive stars $(\sim 20 \%)$ mixes with ISM $(\sim 80 \%)$ and is accelerated by subsequent supernovae.

Recent SuperTIGER analysis has extended the UHGCR measurements from the first flight up to ${ }_{56} \mathrm{Ba}[3,4]$. The preliminary GCRS abundances above ${ }_{40} \mathrm{Zr}$ in Fig. 5 show volatile elements are enhanced to roughly the same degree as the refractories. The breakdown of the OB association GCRS model for UHGCR elements where the s-process no longer dominates over the r-process suggests the possibility of another source component other than ISM and fresh massive star material, such as neutron star merger (NSM) material. The breakdown in the preferential acceleration of refractory elements may also in part result from this material being old enough that the volatile elements have had sufficient time and appropriate conditions to condense onto dust grains at a level comparable to the refractory elements.

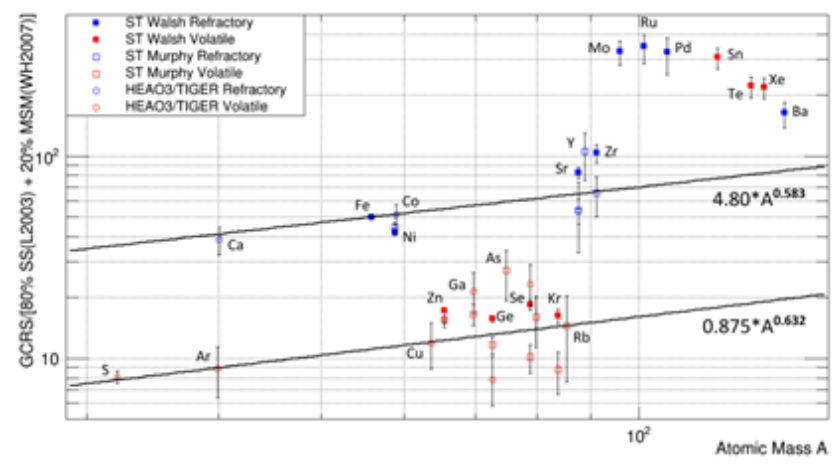

Figure 4(a): SuperTIGER results through ${ }_{56} \mathrm{Ba}$ showing that existing model is insufficient for elements above $40 \mathrm{Zn}$.

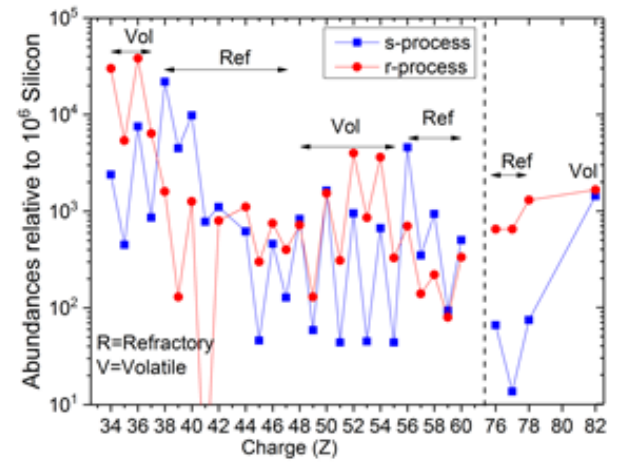

Figure 4(b): Model of s- and r-process composition showing refractory and volatile elements.

TIGERISS observations could determine the relative amounts of nucleosynthesis by s- and r-processes neutron capture in the UHGCR as a function of charge. Observations through ${ }_{40} \mathrm{Zr}$ have shown that the UHGCR are significantly enhanced in the s-process neutron capture products from massive stars, and in measuring heavier elements TIGERISS will also be able to measure r-process neutron capture products to probe for signatures of contributions from massive stars and NSM. Figure $4 \mathrm{~b}$ gives a model deconvolution of the two neutron-capture processes as a function of atomic number that also shows which elements are volatile and refractory. To fully disentangle the contributions from GCRS components and preferential acceleration will require measuring UHGCR abundances through ${ }_{82} \mathrm{~Pb}$, which is a long term goal of TIGERISS and will help constrain models of NSM contributions to the GCR. However, in only one year significant measurements could be made of the more abundant dominant s-process $\left({ }_{50} \mathrm{Sn}\right.$ and $\left.{ }_{56} \mathrm{Ba}\right)$ and r-process elements $\left({ }_{52} \mathrm{Te},{ }_{54} \mathrm{Xe}\right)$. Three of these are volatile $\left({ }_{50} \mathrm{Sn},{ }_{52} \mathrm{Te}\right.$ and $\left.{ }_{54} \mathrm{Xe}\right)$ and only one is refractory $\left({ }_{56} \mathrm{Ba}\right)$, which allows us to disentangle the impact of acceleration efficiency to determine the relative contributions of the $\mathrm{r}$ - 
and s-process to the GCRS in the ${ }_{50} \mathrm{Sn}-{ }_{56} \mathrm{Ba}$ group.

\subsection{Energy Spectra}

TIGERISS will be able to measure the energy spectra of the more abundant elements between ${ }_{5} \mathrm{~B}$ to ${ }_{28} \mathrm{Ni}$ from above the $\sim 200 \mathrm{MeV} /$ nucleon detection threshold to where the aerogel Cherenkov signal plateaus, near $\beta=1$ above $\sim 10 \mathrm{GeV} /$ nucleon. These measurements will complement those by other instruments, including AMS-02 and CALET, already on the ISS, and the ACE-CRIS and DAMPE satellite instruments. The comparatively large area of TIGERISS will make it a sensitive probe for temporarily variant spectral features that could arise from phenomena like microquasars.

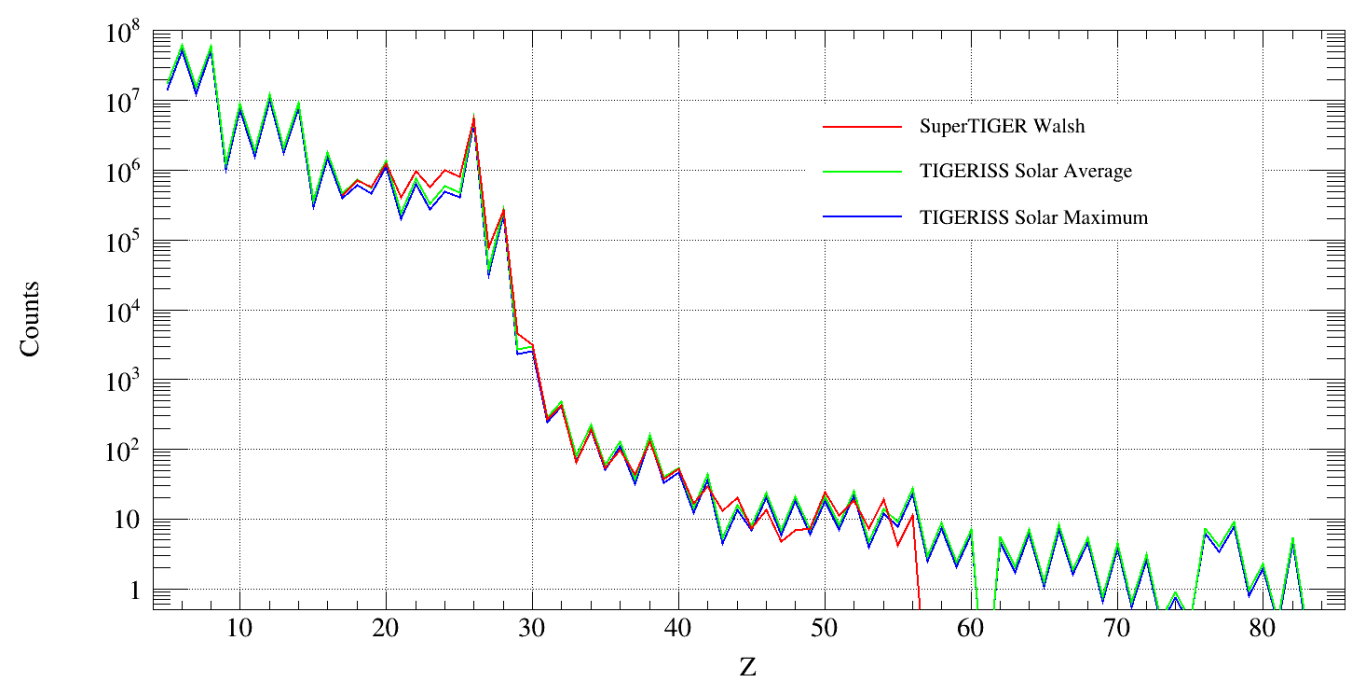

Figure 5: Predicted abundances measured by TIGERISS after 1 year of operation are comparable to those measured by SuperTIGER over its 55 day long-duration-balloon flight [3, 4].

\section{Predicted TIGERISS Observations}

\subsection{One Year Statistics for Astrophysics Pioneers}

TIGERISS would be able to address important science goals in the $\sim 1$ year it would have on ISS under the NASA Astrophysics Pioneers Program, in which time it would also make the case for continuing operations under other funding. Figure 5 gives predicted one year TIGERISS measurements for the JEM-EF configuration [8] for solar minimum (blue) and solar average (green) modulations compared with those from the first SuperTIGER flight (red) [3, 4]. The level of solar modulation does not have a strong impact on the TIGERISS UHGCR measurements, and the expected one-year statistics are comparable to SuperTIGER. The TIGERISS predictions above ${ }_{40} \mathrm{Zr}$ are based on the assumed $80 \%$ even $/ 20 \%$ odd splitting of the even-odd pairs measured by HEAO-3-C2 [18], which agree reasonably with the SuperTIGER abundances. Notable differences are unstable ${ }_{43} \mathrm{Tc}$ that has greater secondary production at balloon altitudes and the elements around ${ }_{48} \mathrm{Cd}$ were the transition between high- and low-gain measurements in SuperTIGER makes their measurement suspect. The one-year TIGERSS measurements would have comparable UHGCR 
statistics with superior charge resolution that would check the SuperTIGER results through ${ }_{56} \mathrm{Ba}$. With its superior charge resolution, TIGERISS would also be able to probe the $r$ - and s-process contributions to the UHGCR in the ${ }_{50} \mathrm{Sn}-{ }_{56} \mathrm{Ba}$ group.

\subsection{Statistics from Extended Observations}

After the successful demonstration of TIGERISS in the first year of operations, we would propose extended operations in order to increase its scientific return. Figure 6 shows the TIGERISS statistics expected under average solar modulation conditions from five years of observations with statistical error bars and a line showing that most even elements will be measured with $20 \%$ statistical precision or better, including ${ }_{76} \mathrm{Os},{ }_{78} \mathrm{Pt}$ and ${ }_{82} \mathrm{~Pb}$. The excellent charge resolution of TIGERISS will yield significant measurements of even the rarer elements with lower statistical precision. Extended observations would also allow comparison of $\mathrm{r}$ - and s-process contributions to the

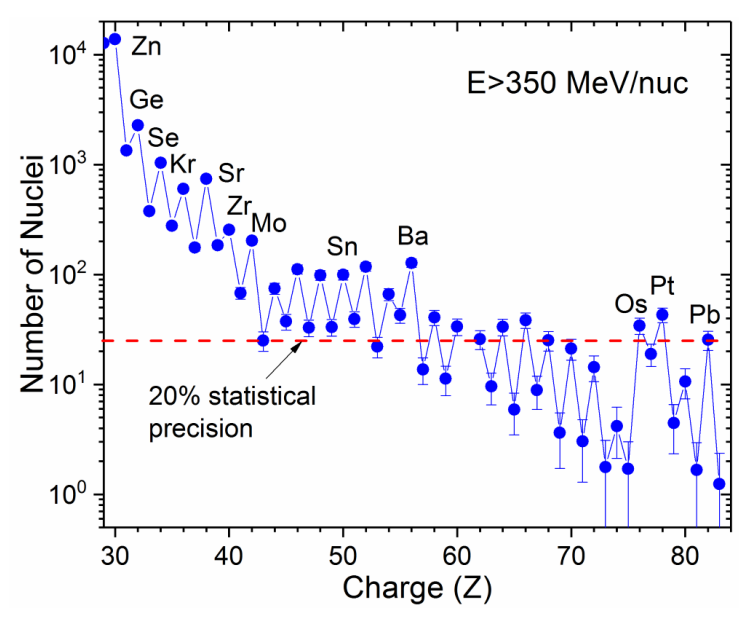

Figure 6: Predicted abundances measured by TIGERISS after 5 years of operation.

UHGCR, as well as to search for any r-process enhancement as a function of atomic number that might indicate an additional source such as NSM.

\section{Conclusions}

The TIGERISS instrument combining silicon strip and Cherenkov detectors will be capable of measuring all of the GCR from ${ }_{5} \mathrm{~B}$ to ${ }_{82} \mathrm{~Pb}$ with single element resolution, which will probe the GCRS and the mechanism that injects this material into the accelerator. In one-year of measurements under the Astrophysics Pioneers Program, TIGERISS will have different systematics that will verify the SuperTIGER results and double the statistics of this UHGCR measurement that conflicts with the leading GCRS model. TIGERISS will also make the first preliminary single element UHGCR measurements from ${ }_{56} \mathrm{Ba}$ to ${ }_{82} \mathrm{~Pb}$ while probing the relative contributions of $\mathrm{r}$ - and s-process neutron capture to the GCRS in the ${ }_{50} \mathrm{Sn}-{ }_{56} \mathrm{Ba}$ group.

\section{References}

[1] B. P. Abbott et al., "Gw170817: Observation of gravitational waves from a binary neutron star inspiral," Phys. Rev. Lett., vol. 119, p. 161101, Oct 2017.

[2] B. P. Abbott et al., "Multi-messenger observations of a binary neutron star merger," The Astrophysical Journal, vol. 848, p. L12, oct 2017.

[3] N. E. Walsh, SuperTIGER Elemental Abundances for the Charge Range $41 \leq Z \leq 56$. PhD thesis, Washington University in St. Louis, 2020. 
[4] N. E. Walsh et al., "SuperTIGER Abundances of Galactic Cosmic Rays for the Atomic Number (Z) Interval 30 to 56," in Proceedings of 37th International Cosmic Ray Conference - PoS(ICRC2021), vol. 395, p. 118, 2021.

[5] J. F. Krizmanic et al., "HNX/SuperTIGER Silicon Strip Detector Response to Nuclei in Lead Primary and Fragmented Test Beams," in 36th International Cosmic Ray Conference (ICRC2019), vol. 36 of International Cosmic Ray Conference, p. 94, July 2019.

[6] B. F. Rauch et al., "Cosmic Ray Origin in OB Associations and Preferential Acceleration of Refractory Elements: Evidence from Abundances of Elements ${ }_{26} \mathrm{Fe}$ through ${ }_{34} \mathrm{Se}$," The Astrophysical Journal, vol. 697, no. 2, pp. 2083-2088, 2009.

[7] R. P. Murphy et al., "Galactic Cosmic Rays Origins and OB Associations: Evidence from SuperTIGER Observations of Elements ${ }_{26} \mathrm{Fe}$ through ${ }_{40} \mathrm{Zr}$," The Astrophysical Journal, vol. 831, no. 2, p. 148, 2016.

[8] B. F. Rauch et al., "Determination of Expected TIGERISS Observations," in Proceedings of 37th International Cosmic Ray Conference - PoS(ICRC2021), vol. 395, p. 088, 2021.

[9] W. V. Zober et al., "Determination of Expected TIGERISS Observations," in Proceedings of 37th International Cosmic Ray Conference - PoS(ICRC2021), vol. 395, p. 124, 2021.

[10] K. Lodders, "Solar System Abundances and Condensation Temperatures of the Elements," The Astrophysical Journal, vol. 519, pp. 1220-1247, 2003.

[11] T. Sanuki et al., "Precise Measurement of Cosmic-Ray Proton and Helium Spectra with the BESS Spectrometer," The Astrophysical Journal, vol. 545, no. 2, pp. 1135-1142, 2000.

[12] M. Aguilar et al., "Isotopic Composition of Light Nuclei in Cosmic Rays: Results from AMS-01," The Astrophysical Journal, vol. 736, p. 105, Aug. 2011.

[13] J. J. Engelmann et al., "Charge Composition and Energy Spectra of Cosmic-Ray Nuclei for Elements from Be to Ni. Results from HEAO-3-C2," Astronomy \& Astrophysics, vol. 233, pp. 96-111, 1990.

[14] W. R. Binns et al., "Elemental Source Composition Measurements and the Origin of Galactic Cosmic Rays," in 36th International Cosmic Ray Conference (ICRC2019), vol. 36 of International Cosmic Ray Conference, p. 36, July 2019.

[15] J. C. Higdon and R. E. Lingenfelter, "OB Associations, Supernova-Generated Superbubbles, and the Source of Cosmic Rays," The Astrophysical Journal, vol. 628, pp. 738-749, 2005.

[16] W. R. Binns et al., "Observation of the ${ }^{60} \mathrm{Fe}$ nucleosynthesis-clock isotope in galactic cosmic rays," Science, vol. 352, pp. 677-680, May 2016.

[17] R. E. Lingenfelter, “The Origin of Cosmic Rays: How Their Composition Defines Their Source and Sites and the Process of Their Mixing, Injection, and Acceleration," The Astrophysical Journal Supplement Series, vol. 245, p. 30, 2019.

[18] W. R. Binns et al., "Abundances of Ultraheavy Elements in the Cosmic Radiation: Results from HEAO 3,” The Astrophysical Journal, vol. 346, pp. 997-1009, 1989. 


\section{Full Authors List: TIGERISS Collaboration}

M. Alfred ${ }^{1}$, M. Amoo ${ }^{2}$, T. Anderson ${ }^{3}$, R. G. Bose ${ }^{4}$, T. J. Brandt ${ }^{5}$, J. H. Buckley ${ }^{4}$, N. Cannady,7,8, R. Caputo ${ }^{5,7}$, S. Coutu ${ }^{3}$, C. Kierans ${ }^{5,7}$, J. F. Krizmanic ${ }^{6,7,8}$, A. W. Labrador ${ }^{9}$, J. V. Martins ${ }^{10}$, J. G. Mitchell ${ }^{11,12}$, J. W. Mitchell ${ }^{7}$, S. A. I. Mognet ${ }^{3}$, A. A. Moiseev ${ }^{7,8,13}$, G. A. de Nolfo $^{12}$, S. Nutter ${ }^{14}$, B. F. Rauch ${ }^{4}$, K. Sakai ${ }^{6,7,8}$, M. Sasaki ${ }^{7,8,13}$, N. E. Walsh ${ }^{4}$, L. P. Williams ${ }^{6,7,8}$, and W. V. Zober ${ }^{4}$

${ }^{1}$ Department of Electrical Engineering and Computer Science, Howard University, Washington, DC 20059, USA

${ }^{2}$ Department of Physics and Astronomy, Howard University, Washington, DC 20059, USA

${ }^{3}$ Department of Physics, Penn State University, University Park, PA 16802, USA

${ }^{4}$ Department of Physics and McDonnell Center for the Space Sciences, Washington University, St. Louis, MO 631304899, USA

${ }^{5}$ NASA Goddard Space Flight Center, Greenbelt, MD 20771, USA

${ }^{6}$ Center for Space Sciences and Technology, University of Maryland Baltimore County, Baltimore, MD 21250, USA

${ }^{7}$ Astroparticle Physics Laboratory, NASA/GSFC, Greenbelt, Maryland 20771, USA

${ }^{8}$ Center for Research and Exploration in Space Sciences and Technology, NASA/GSFC, Greenbelt, MD 20771, USA

${ }^{9}$ California Institute of Technology, Pasadena, CA 91125, USA

${ }^{10}$ Department of Physics, University of Maryland Baltimore County, Baltimore, MD 21250, USA

${ }^{11}$ Department of Physics, The George Washington University, Washington, DC 20052, USA

${ }^{12}$ Heliospheric Physics Laboratory, NASA/GSFC, Greenbelt, MD 20771, USA

${ }^{13}$ Department of Astronomy, University of Maryland, College Park, MD 20742, USA

${ }^{14}$ Dept. of Physics and Geology, Northern Kentucky University, Newport, KY 41099, USA 